\title{
Bee venom stimulates human melanocyte proliferation, melanogenesis, dendricity and migration
}

\author{
Songhee Jeon ${ }^{1 *}$, Nan-Hyung $\mathrm{Kim}^{2 *}$, \\ Byung-Soo Koo ${ }^{3}$, Hyun-Joo Lee ${ }^{2}$ and \\ Ai-Young Lee ${ }^{1,4}$ \\ ${ }^{1}$ Dongguk University Research Institute of Biotechnology \\ Medical Science Research Center \\ Goyang 410-773, Korea \\ ${ }^{2}$ Department of Dermatology \\ Dongguk University School of Medicine \\ Goyang 410-773, Korea \\ ${ }^{3}$ Department of Neuropsychiatry \\ College of Oriental Medicine \\ Dongguk University \\ Goyang 410-773, Korea \\ ${ }^{4}$ Corresponding author: Tel, 82-31-961-7250; \\ Fax, 82-31-961-7695; E-mail, leeay@ duih.org \\ *These authors contributed equally to this work.
}

Accepted 31 July 2007

Abbreviations: Akt, protein kinase $B$; CREB, cAMP response element binding protein; PI3K, phosphatidylinositol 3-kinase; $\mathrm{SPLA}_{2}$, secreted phospholipase $A_{2}$

\begin{abstract}
Pigmentation may result from melanocyte proliferation, melanogenesis, migration or increases in dendricity. Recently, it has been reported that secreted phospholipase $A_{2}\left(S P L A_{2}\right)$ known as a component of bee venom (BV), stimulates melanocyte dendricity and pigmentation. BV has been used clinically to control rheumatoid arthritis and to ameliorate pain via its anti-inflammatory and antinociceptive properties. Moreover, after treatment with BV, pigmentation around the injection sites was occasionally observed and the pigmentation lasted a few months. However, no study has been done about the effect of BV on melanocytes. Thus, in the present study, we examined the effect of $\mathrm{BV}$ on the proliferation, melanogenesis, dendricity and migration in normal human melanocytes and its signal transduction. BV increased the number of melanocytes dose and time dependently through PKA, ERK, and PI3K/Akt activation. The level of CAMP was also increased by BV treatment. Moreover, BV induced melanogenesis through increased tyrosinase expression. Furthermore, BV induced melanocyte dendricity and
\end{abstract}

migration through $\mathrm{PLA}_{2}$ activation. Overall, in this study, we demonstrated that BV may have an effect on the melanocyte proliferation, melanogenesis, dendricity and migration through complex signaling pathways in vitro, responsible for the pigmentation. Thus, our study suggests a possibility that BV may be developed as a therapeutic drug for inducing repigmentation in vitiligo skin.

Keywords: bee venoms; cell movement; cell proliferation; melanocytes; melanins; skin pigmentation; vitiligo

\section{Introduction}

Normal human melanocytes are located in the basal layer of the epidermis and rarely undergo mitosis (Jimbow et al., 1975; Pawelek, 1979). It is well accepted that paracrine growth factors produced by keratinocytes, or growth-promoting agents, such as endothelin-1 (ET-1), stem cell factor (SCF), basic fibroblast growth factor (bFGF), 12-Otetradecanoylphorbol 13-acetate (TPA), cAMPelevating agents (forskolin, IBMX, $\alpha-M S H$ ) and others, regulate melanocyte proliferation along with other diverse melanocyte functions such as melanin synthesis and dendricity (Hirobe et al., 2004; Imokawa, 2004). The signaling mechanism of each factor in vitro is different. TPA acts on melanocyte growth through activation of PKC (Arita et al., 1992), whereas ET-1 and bFGF may affect melanocyte growth through phosphorylation of ERK 1/2 (Swope et al., 1995; Tada et al., 2002). Activation of ERK1/2 is critical for the mitogenic response of melanocytes. Failure to activate this kinase is evident in terminally differentiated melanocytes (Medrano et al., 1994). In human melanocytes, activation of ERK $1 / 2$ is sequentially connected to CAMP response element binding protein (CREB) activation (Tada et al., 2002). CREB could be a common downstream target for melanocyte differentiation and proliferation.

Pigmentation may result from melanocyte proliferation, melanogenesis, migration (Lan et al., 2005) or increases in dendricity (Scott et al., 2006). Vitiligo is an acquired depigmentation disorder caused by melanocyte death (Kovac, 1998). Although more than $1 \%$ of the general population suffer from vitiligo, recovery of pigmentation is 
often difficult mainly due to the lack of effective methods for treatment. Although several factors, including SCF (Grabbe et al., 1994), bFGF (Halaban et al., 1987), bovine pituitary extract (Wilkins et al., 1985), TPA (Krasagakis et al., 1993), and cAMP stimulating agents, are known to induce the proliferation of melanocytes in vitro, these mitogens are rarely used clinically.

Recently, it has been reported that secreted phospholipase $A_{2}\left(s P L A_{2}\right)$ known as a component of bee venom (BV), stimulates melanocyte dendricity and pigmentation (Maeda et al., 1996; Maeda and Naganuma, 1997; Scott et al., 2006). $\mathrm{BV}$ has been used clinically to control rheumatoid arthritis and to ameliorate pain via its antiinflammatory and antinociceptive properties (Kwon et al., 2001). BV also has been shown to have antibacterial effects (Eiseman et al., 1982; Akdis et al., 1996; Kwon et al., 2001), and has been used as a meridian therapy in Korea (Kim et al., 2003; Lee et al., 2004). Moreover, after treatment with $\mathrm{BV}$, pigmentation around the injection sites was occasionally observed and the pigmentation lasted a few months (unpublished observation), suggesting that BV may have an effect on the melanocyte proliferation, melanogenesis, dendricity or migration responsible for the pigmentation. Thus, in the present study, we examined the effect of BV on the proliferation, melanogenesis, dendricity and migration in normal human melanocytes and elucidated the signaling pathway responsible for the effect of BV.

\section{Materials and Methods}

\section{Normal human epidermal melanocyte culture}

Skin specimens obtained from repeated cesarean sections and circumcisions were used for cultures. Epidermis was separated from dermis after treatment with $2.4 \mathrm{U} / \mathrm{ml}$ of dispase (Roche, Mannheim, Germany) for $1 \mathrm{~h}$. The epidermal sheets were treated with $0.05 \%$ trypsin for $10 \mathrm{~min}$ to produce a suspension of individual epidermal cells. The cells were suspended in Medium 254 (Cascade Biologics, Portland, OR) supplemented with bovine pituitary extract, FBS, bovine insulin, hydrocortisone, bFGF, and bovine transferrin (Cascade Biologics, Portland, OR), heparin, and PMA (Cascade Biologics, Portland, OR). When the cells reached confluence, they were detached from the flask and seeded into other culture flasks.

\section{Treatment of melanocytes with BV or forskolin with/without inhibitors}

Melanocytes from passage numbers between 7 and 15 were used for the experiments. Medium 254 with supplements was changed to medium containing $1 / 5$ the amount of supplements for $24 \mathrm{~h}$ before treatment with BV (GUJU Pharma CO., Seoul, Korea). To determine the appropriate concentration of $\mathrm{BV}$, dose-response studies using various concentrations between 1.25 and $20 \mu \mathrm{g} / \mathrm{ml}$ were done. Then, the cells were treated with 10 $\mu \mathrm{g} / \mathrm{ml} \mathrm{BV}$ or $10 \mu \mathrm{M}$ forskolin for $1,3,5$ or 7 days (Calbiochem, La Jolla, CA). Five cell lines were examined.

For the inhibition experiments, melanocytes were incubated in the media with $1 / 5$ supplements, with addition of $2.5 \mu \mathrm{M}$ U0126, an MEK inhibitor, 1 $\mu \mathrm{M}$ wortmannin (Calbiochem, La Jolla, CA), a PI3K inhibitor, or $10 \mu \mathrm{M} \mathrm{H} 89$ (Calbiochem, Jolla, $\mathrm{CA})$, a PKA inhibitor, for $60 \mathrm{~min}$ before BV treatment.

\section{MTT assay}

The proliferation of melanocytes was examined using an MTT assay kit (R\&D Systems, Minneapolis, MN). Each absorbance was measured at a spectrum of $570 \mathrm{~nm}$. The MTT assay was conducted with melanocytes treated with BV in the absence or presence of the above-mentioned stimulators or inhibitors

\section{Measurement of melanin content}

The melanin content of cultured melanocytes was measured in accordance with the method described by Oka et al. (1996). Briefly, the cell pellets were solubilized in boiled $1 \mathrm{M} \mathrm{NaOH}\left(80^{\circ} \mathrm{C}\right)$ for $2 \mathrm{~h}$. The color was analyzed at a spectrum of $405 \mathrm{~nm}$. To examine the true melanin formation from the same number of cells, the total melanin content of each pellet was divided by the number of melanocytes.

\section{RT-PCR}

Total RNA from melanocytes cultured under each condition was isolated using the QuickGene RNA cultured cell HC kit (Life Science, Minato-ku, Tokyo, Japan). cDNA was synthesized from total RNA using the First Strand cDNA Synthesis Kit for RT-PCR (AMV; Boehringer Mannheim, Germany). Human GAPDH was used as the internal standard. The primer sequences of MITF were: forward 5'ATGCTGGAAATGCTAGAATATAAT-3'; and reverse 5'-ATCATCCATCTGCATACAG-3'; and the primer 
sequences of GAPDH were: forward 5'-TCCACTGGCGTCTTCACC-3'; and reverse 5'-GGCAGAGATGATGACCCTTTT-3'. PCR amplification was conducted in a $40 \mu \mathrm{l}$ reaction, consisting of $10 \times$ reaction buffer, $2.5 \mathrm{mM} \mathrm{MgCl}_{2}, 250 \mu \mathrm{M}$ dNTPs, $100 \mathrm{ng}$ each PCR primer, $0.5 \cup$ Taq DNA polymerase, and 20-50 ng DNA, using a DNA Thermal Cycler 9600 (Applied Biosystems, Foster city, CA) at $94^{\circ} \mathrm{C}$ for 1 min, $58^{\circ} \mathrm{C}$ for $1 \mathrm{~min}$, and $72^{\circ} \mathrm{C}$ for $1 \mathrm{~min}$ at 30 cycles. The DNA fragments produced by PCR were separated by electrophoresis on a $2 \%$ agarose gel.

\section{cAMP immunoassay}

Melanocytes were lysed in $0.1 \mathrm{M} \mathrm{HCl}$ to inhibit phosphodiesterase activity and centrifuged at $2,000 \mathrm{~g}$ for $15 \mathrm{~min}$. The concentration of cAMP was measured using the cAMP assay kit in accordance with the manufactures instructions (Biomol International, Plymouth, PA) and expressed as pg per $\mathrm{ml}$ per $1 \times 10^{5}$.

\section{Western blot analysis}

The cultured melanocytes treated with BV or forskolin with or without inhibitor were homogenized in ice-cold homogenization buffer containing $50 \mathrm{mM}$ Tris-base ( $\mathrm{pH} 7.4), 150 \mathrm{mM} \mathrm{NaCl}, 10$ $\mathrm{mM}$ EDTA, $0.1 \%$ Tween-20, and protease inhibitors $(0.1 \mathrm{mM}$ PMSF, $5 \mu \mathrm{g} / \mathrm{ml}$ aprotinin, and 5 $\mu \mathrm{g} / \mathrm{ml}$ leupeptin). Equal amounts of extracted proteins $(30 \mu \mathrm{g})$ were resolved using $10 \%$ SDSPAGE and transferred to nitrocellulose membranes. The membranes were incubated with antiphospho-MAPK, MAPK, phospho-Akt, Akt, CREB, phospho-CREB (rabbit polyclonal; Cell Signaling Technology, Beverly, MA), MITF (mouse monoclonal; Abcam, Cambridge, UK) or tyrosinase (rabbit polyclonal; Santa Cruz Biotechnology, Santa Cruz, CA) antibody, diluted 1:1000 in blocking solution, overnight at $4^{\circ} \mathrm{C}$. The membranes were further incubated with anti-rabbit or anti-mouse HRP-conjugated antibody (Santa Cruz Biotechnology, Santa Cruz, CA) and treated with an enhanced chemiluminescence solution (Pierce, Rockford, IL). The signals were captured on an Image Reader (LAS-3000; Fuji Photo Film, Tokyo, Japan). To monitor the amount of protein loaded into each lane, the membranes were reprobed with mouse monoclonal anti-actin antibody (Sigma, St. Louis, MO) and processed as described above. The protein bands were analyzed by densitometry.

\section{Cell migration assay}

Cell migration assays were performed as described previously (Park et al., 2005). Cells were allowed to form a confluent monolayer in a 6-well plate. The wound was made by scraping a conventional pipette tip across the monolayer. The width of scratch was measured at randomly selected 10 areas and the meam value was calculated.

\section{Boyden chamber cell migration assay}

Nucleopore filters with $8 \mu \mathrm{m}$ pore size (Corning Inc., Corning, NY) were coated with type I collagen. The human melanocytes $\left(1 \times 10^{4}\right)$ were added to the upper chamber and low chambers were filled with $10 \mu \mathrm{g} / \mathrm{ml}$ BV with/without $\mathrm{PLA}_{2}$ inhibitor. After $72 \mathrm{~h}$ of incubation, the filters were stained with Hoechst and cells on the upper side of the insert were removed with a cotton swab. Three randomly selected fields were photographed, and the cells that had migrated were counted. The migration was expressed as a percentage of migrated cells in positive control melanocytes.

\section{Melanocyte dendricity assay}

Melnocytes were subcultured in 6 well plate $(4 \times$ $10^{5}$ cells $/$ well) and were treated with $10 \mu \mathrm{g} / \mathrm{ml}$ of $\mathrm{BV}$ with/without $\mathrm{PLA}_{2}$ inhibitor for $24 \mathrm{~h}$. Following treatment, the image of cells was obtained using an inverted microscope (DMI 600DB; Leica, Wetzlar, Germany). Dendrites-per cell was quantitated manually from the photographs.

\section{Statistical analysis}

Statistical significance was tested with Student $t$-test. All results are presented as the mean \pm S.E. of the combined data from replicate experiments.

\section{Results}

\section{BV induces melanocyte proliferation and melanogenesis}

To examine whether BV induces melanocyte proliferation dose dependently, 1.25 to $20 \mu \mathrm{g} / \mathrm{ml}$ of BV was treated in human melanocytes for 5 days. As shown in Figure $1 \mathrm{~A}$, at concentrations of $10 \mu \mathrm{g} / \mathrm{ml}$ or higher, BV induced an approximately 1.8 -fold increase in the number of melanocytes, compared to the control. To compare the effect of BV on the melanocyte proliferation, we used forskolin, a CAMP elevating agent. As expected, forskolin treatment also resulted in an increase in the 
A

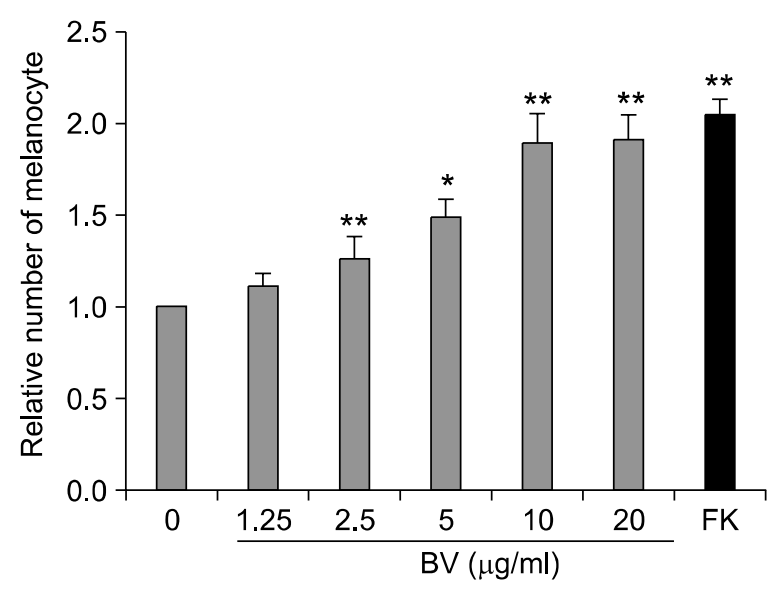

C

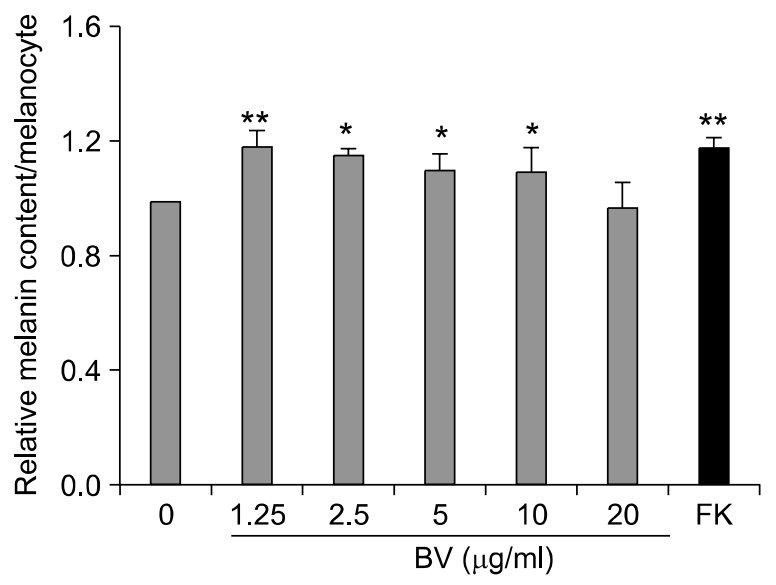

number of melanocytes to a similar extent (Figure $1 \mathrm{~A})$. Then, we examined whether BV could increase the number of normal human melanocytes up to a long time. The proliferation of melanocytes was not remarkable until 3 days after the culture, but getting increased with the time course. The melanocyte proliferation by BV treatment reached approximately 2 times more compared with control in 7 days, which showed similar pattern with that by forskolin treatment (Figure 1B).

To observe whether BV induces melanogenesis in normal melanocytes, the melanin content of individual melanocytes was examined. The melanin content was maximally increased at the lowest dose $(1.25 \mu \mathrm{g} / \mathrm{ml})$ of $B V$ and gradually decreased to $20 \mu \mathrm{g} / \mathrm{ml}$ of $\mathrm{BV}$ in melanocytes (Figure 1C). The effect of melanogenesis of $\mathrm{BV}$ at $1.25 \mu \mathrm{g} / \mathrm{ml}$ was similar with that of forskolin (Figure 1C). However, at $20 \mu \mathrm{g} / \mathrm{ml}$ of BV reduced melanin

\section{B}

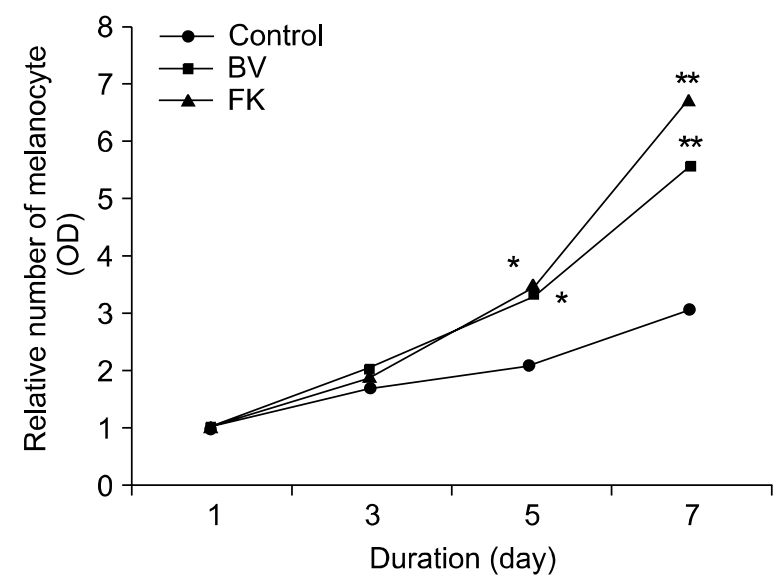

Figure 1. Bee venom (BV) induces melanocyte proliferation and melanogenesis. (A) Supplement-reduced melanocytes were treated with $0-20 \mu \mathrm{g} / \mathrm{ml} \mathrm{BV}$ or $10 \mu \mathrm{M}$ forskolin (FK). The number of melanocytes was examined $5 \mathrm{~d}$ after BV treatment using the MTT assay. The number of melanocytes increased dose dependently up to $10 \mu \mathrm{g} / \mathrm{ml}$ BV. The error bars indicate S.E. $(n=6) ;{ }^{*} P<0.01 ;{ }^{* *} P<0.001$. (B) The number of melanocytes was examined on days $1,3,5$, and 7 after $10 \mu \mathrm{g} / \mathrm{ml}$ of BV treatment or $10 \mu \mathrm{M}$ of forskolin (FK) by using MTT assay. The error bars indicate S.E. $(n=3){ }^{*} P<0.01,{ }^{* *} P<0.001$. (C) Melanin content was measured $5 \mathrm{~d}$ after BV or forskolin treatment. The melanin contents were slightly increased up to $10 \mu \mathrm{g} / \mathrm{ml}$ of BV. The error bars indicate S.E. $(n=3) ;{ }^{*} P<0.01,{ }^{* *} P<0.001$.

content compared to that of control. These results indicate that the effect of BV may be different in accordance with dosage in mealnocytes; at high dose of BV induces melanocyte proliferation, while at low dose of BV induces melanocyte differentiation. However, at $10 \mu \mathrm{g} / \mathrm{ml}$, BV also significantly increased melanocyte proliferation as well as melanin content compared to that of control. Since melanocyte proliferation rather than melanogenesis is considered as an important factor to induce repigmentation in depigmented skin, other effects of $\mathrm{BV}$ were also examined at the concentration of $10 \mu \mathrm{g} / \mathrm{ml}$ in human melanocytes.

\section{BV increases cAMP levels}

cAMP play key roles in the regulation of skin and hair pigmentation. Moreover, when we compared the effect of BV on the melanocyte proliferation 
Table 1. Effects on the level of cAMP.

\begin{tabular}{cc}
\hline & Concentration of cyclic AMP $(\mathrm{pg} / \mathrm{ml})$ \\
\hline Con & $0.742 \pm 0.084$ \\
BV & $2.456 \pm 0.119$ \\
FK & $29.666 \pm 1.527$ \\
\hline
\end{tabular}

Human melanocytes were incubated with $10 \mu \mathrm{g} / \mathrm{ml} \mathrm{BV}$ or $10 \mu \mathrm{M}$ forskolin for $1 \mathrm{~h}$. The level of CAMP was detected using the CAMP kit (Biomol) and is expressed as concentration per $1 \times 10^{5}$ cells. The values are means \pm S.E. $(n=3)$

and melanogenesis with that of forskolin, the proliferative or melanogenesis effect of BV was similar with that of forskolin. Thus, we checked whether BV induces cAMP levels in the melanocyte. Forskolin is a direct activator of adenylate cyclase (Englaro et al., 1995). Forskolin increased cAMP levels by 40 fold while BV increased the levels by only 3 fold (Table 1). Nevertheless, foskolin and BV promoted the proliferation of melanocytes with the same extent (Figure 1A). These data suggest that BV has other mechanisms to promote melanocyte proliferation though increased cAMP levels may be one of the mechanisms.

\section{BV induces MITF-M and tyrosinase expression in melanocytes}

Previously, we found that $10 \mu \mathrm{g} / \mathrm{ml}$ of BV increased intracellular cAMP level (Table 1) and increased melanin content in melanocytes. Melanins are synthesized in melanosomes that contain the specific enzymens required for proper melanin production. Among them, the most well-characterized are tyrosinase, tyrosinase-related protein 1 (TPR1) and TRP2 (dopachrome tautomerase, DCT) (Ito, 2003). As the molecular events, cAMP activates PKA and simultaneously CREB transcription factor, which leads to upregulate the expression of microphthalmia associated transcription factor M (MITF-M) (Bertolotto et al., 1998), a melanocyte-specific transcription factor crucial for melnocyte development and differentiation (Hodgkinson et al., 1993) and the increased MITF$M$ expression up-regulates tyrosinase expression (Busca and Ballotti, 2000)

Thus, we examined whether BV is able to induce MITF-M and tyrosinase expression in human melanocytes by using RT-PCR and western blot. Following BV treatment, the levels of MITF-M mRNA expression gradually increased until $4 \mathrm{~h}$ (Figure 2A) and also MITF-M protein expression was maximally increased on day 3 and slowly decreased until 5 days (Figure 2B). Furthermore,
A

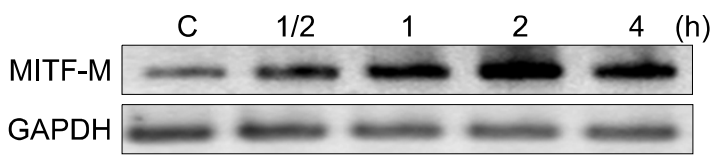

B

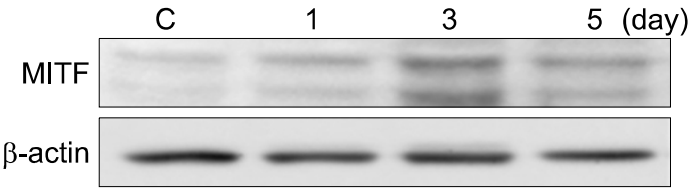

C

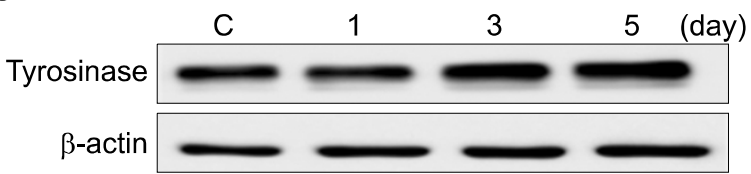

Figure 2. BV induces MITF-M and tyrosinase expression in melanocytes. Supplement-reduced melanocytes were treated with 10 $\mu \mathrm{g} / \mathrm{ml} \mathrm{BV}$ for $0.5,1,2$, or $4 \mathrm{~h}$ and for 1,3 , or $5 \mathrm{~d}$. The whole lysates were electrophoresed in SDS-PAGE and analysed by immunoblotting with each antibody. Data were taken from triplicate experiments with four cell lines. C: non-treated melanocytes (A) Expression of MITF-M mRNA levels increased in supplement-reduced melanocytes treated with $10 \mu \mathrm{g} / \mathrm{ml}$ BV for up to $4 \mathrm{~h}$. (B, C) The expression of MITF and tyrosinase was examined on days 1,3 and 5 after BV treatment.

we examined the expression of tyrosinase by BV treatment. It was gradually increased until 5 days (Figure 2C). These results suggest that high dose of BV $(10 \mu \mathrm{g} / \mathrm{ml})$ also induces proliferation as well as melanogenesis through MITF-M and tyrosinase expression in human melanocyte.

\section{Signaling pathways for BV-induced melanocyte proliferation and tyrosinase expression.}

As previously mentioned, BV increased the level of cAMP in human melanocytes. cAMP stimulating agent increases MITF expression through PKA activation and PKA phosphorylates and activates CREB (Bertolotto et al., 1998). Moreover, cAMP also activates ERK (Englaro et al., 1995). Thus, in order to determine which signaling pathways are activated by BV treatment in human melanocytes, phosphorylation of ERK and CREB was examined using phosphospecific antibodies after treating cells with $10 \mu \mathrm{g} / \mathrm{ml}$ of BV.

The phosphorylation of ERK was increased at $30 \mathrm{~min}$, lasted for 3 days by treatment of BV but decreased on day 5 (Figure 3A, top). CREB phosphorylation also was increased at $30 \mathrm{~min}$ and lasted for 5 days (Figure $3 \mathrm{~A}$, bottom). To verify each signaling pathway, we used specific inhibi- 
A

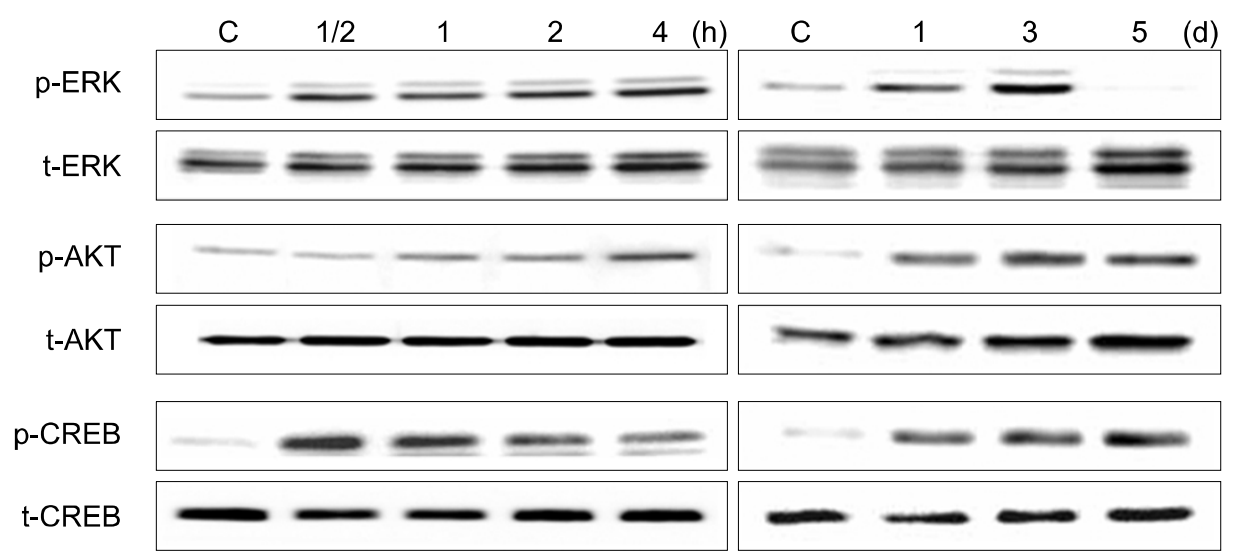

B
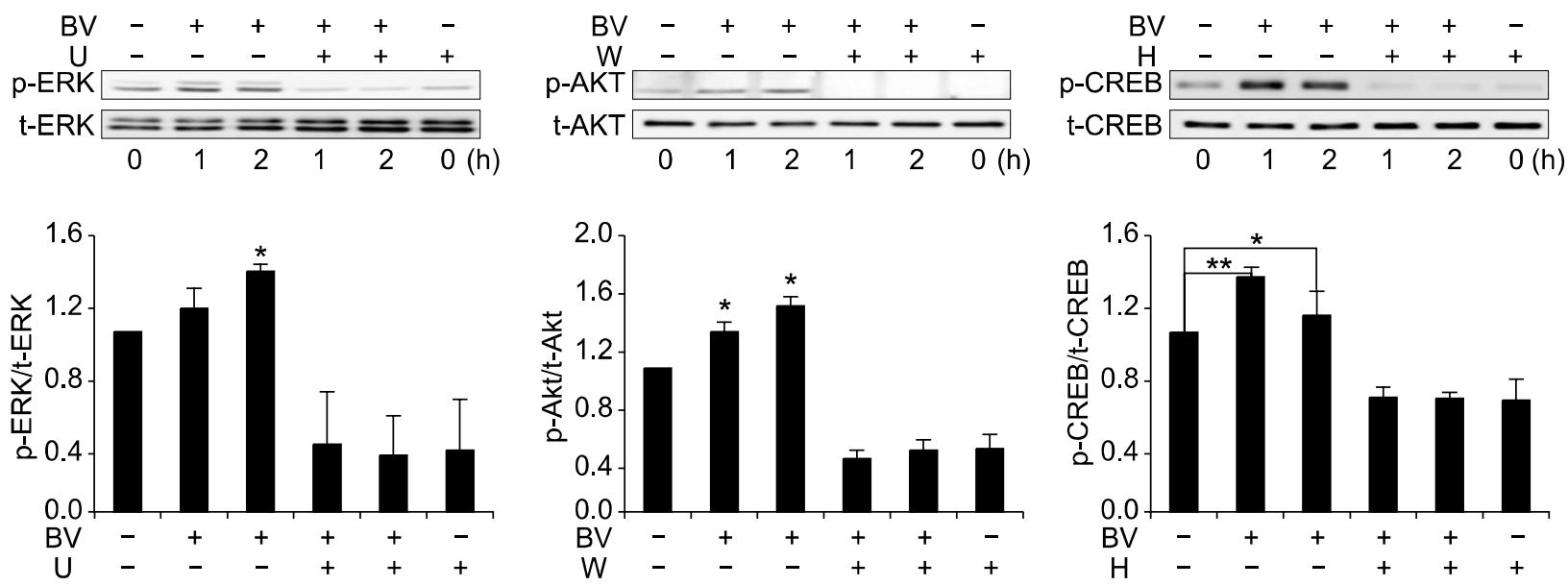

Figure 3. Signaling pathways for BV-induced melanocyte proliferation and tyrosinase expression. (A) Supplement-reduced melanocytes were treated with $10 \mu \mathrm{g} / \mathrm{ml} \mathrm{BV}$ and the cells were lysed at indicated time. The whole lysates were electrophoresed in SDS-PAGE and analyzed by immunoblotting with each antibody. Data were taken from triplicate experiments with four cell lines. C: non-treated melanocytes. (B) For inhibitor experiments, the phosphorylation of ERK, Akt, and CREB were examined $1 \mathrm{~h}$ and $2 \mathrm{~h}$ after BV treatment in the absence or presence of $2.5 \mu \mathrm{M} \mathrm{U} 0126(\mathrm{U}), 1 \mu \mathrm{M}$ wortmannin $(\mathrm{W})$ and $10 \mu \mathrm{M}$ $\mathrm{H} 89 \mathrm{H}$ ). Whole lysates were electrophoresed on SDS-PAGE and analyzed by immunoblotting with each antibody. Data were taken from triplicate experiments with four cell lines. The error bars indicate S.E. $(n=3) ;{ }^{*} P<0.01$; ${ }^{* *} P<0.001$. (C) Supplement-reduced melanocytes were stimulated with 10 $\mu \mathrm{g} / \mathrm{ml} \mathrm{BV}$ for $72 \mathrm{~h}$ in the absence or presence of $2.5 \mu \mathrm{M}$ U0126 (U), $1 \mu \mathrm{M}$ wortmannin $(\mathrm{W})$ or $10 \mu \mathrm{M} \mathrm{H} 89(\mathrm{H})$. The number of melanocytes was examined by using the MTT assay. In the presence of U0126, wortmannin, and H89, BV-induced melanocyte proliferation was inhibited. The error bars indicate S.E. $(n=3) ;{ }^{* *} P<0.001$. (D) Supplement-reduced melanocytes were stimulated with $10 \mu \mathrm{g} / \mathrm{ml} \mathrm{BV}$ for $72 \mathrm{~h}$ in the absence or presence of $2.5 \mu \mathrm{M}$ of $\mathrm{U} 0126$ $(\mathrm{U}), 1 \mu \mathrm{M}$ wortmannin $(\mathrm{W})$ or $10 \mu \mathrm{M} \mathrm{H} 89(\mathrm{H})$. Then, the whole lysates were subjected to western blot analysis. BV-induced tyrosinase expression was completely inhibited by U0126 and H89, whereas wortmannin had no effect. For the control, $\beta$ actin was examined. The error bars indicate S.E. $(n=4)$; ${ }^{*} P<0.01 ;{ }^{* *} P<0.001$.

tors. U0126 is an inhibitor of MEK, which is an upstream kinase of ERK (Robinson and Cobb, 1997). BV-induced ERK phosphorylation was effectively inhibited by pre-treatment of U0126 (Figure 3B, left). To suppress the CREB phosphorylation, we pre-treated H89, a PKA inhibitor in melanocytes. BV-induced CREB phosphorylation was completely suppressed by H89 (Figure 3B, right). However, in the presence of U0126, BVinduced CREB phosphorylation was partially sup- pressed (data not shown), suggesting that BVinduced CREB phosphorylation may be through PKA as well as ERK activation.

Among the signaling pathways putatively involved in melanocyte differentiation and survival, the phosphatidylinositol 3 kinase (PI3K)/Akt pathway seems to be a good candidate, since PI3K has been participated in the differentiation of the neuronal cell model, the PC12 cells (Kimura et al., 1994). In these and subsequent experiments, in 
C

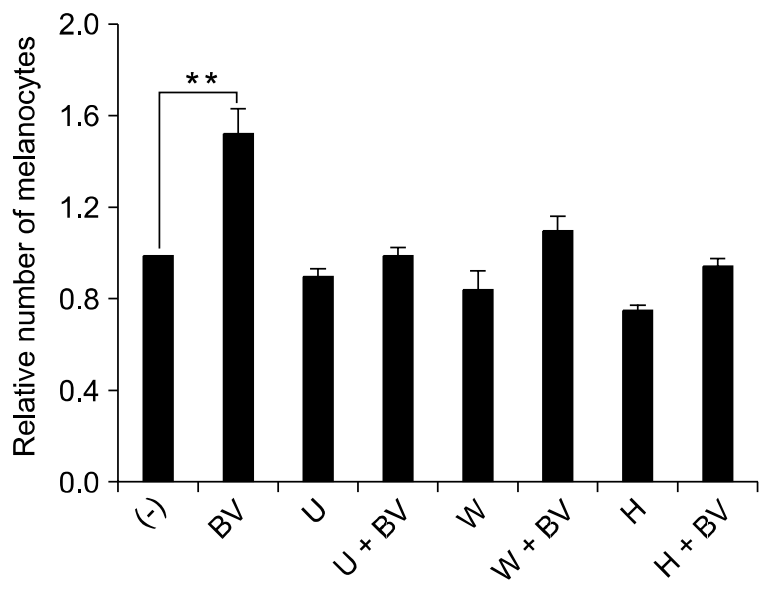

Figure 3. Continued.

D

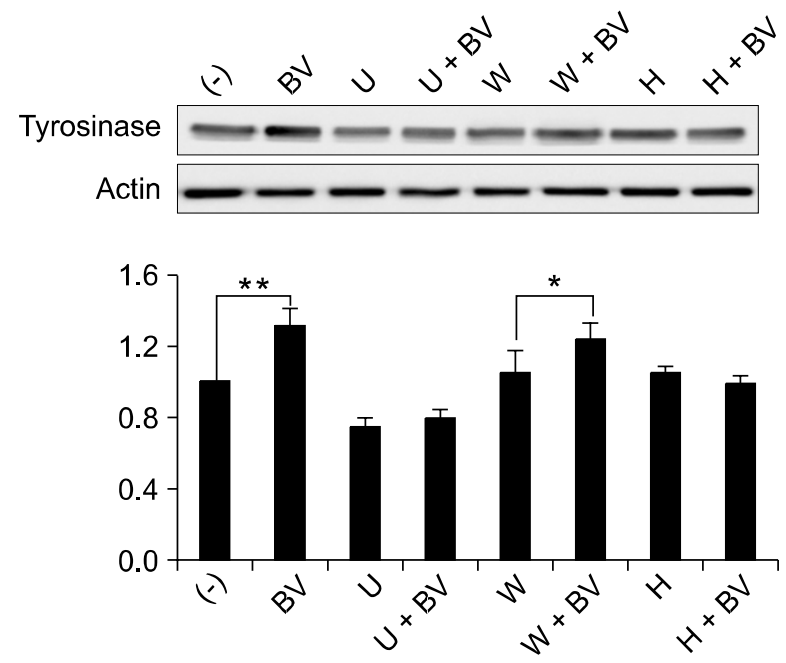

A

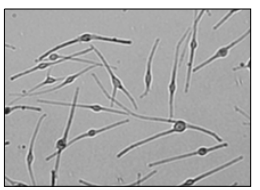

Con

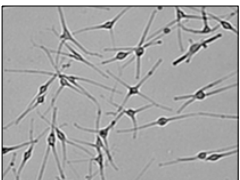

BV

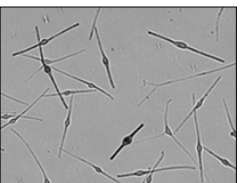

$A+B V$

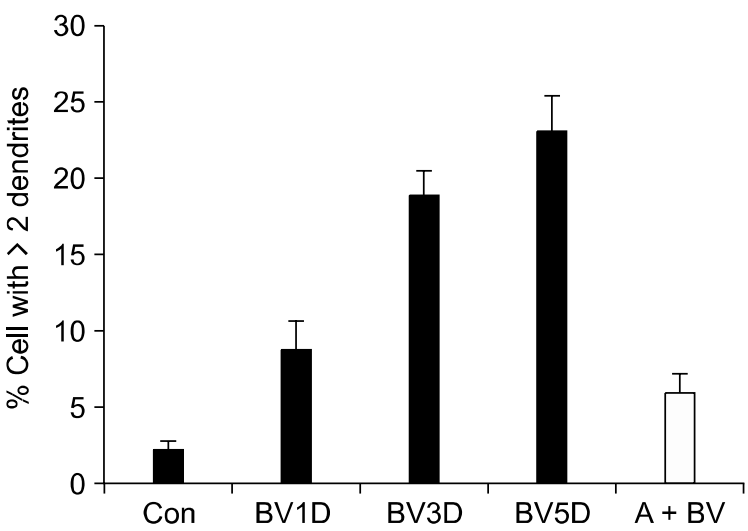

B

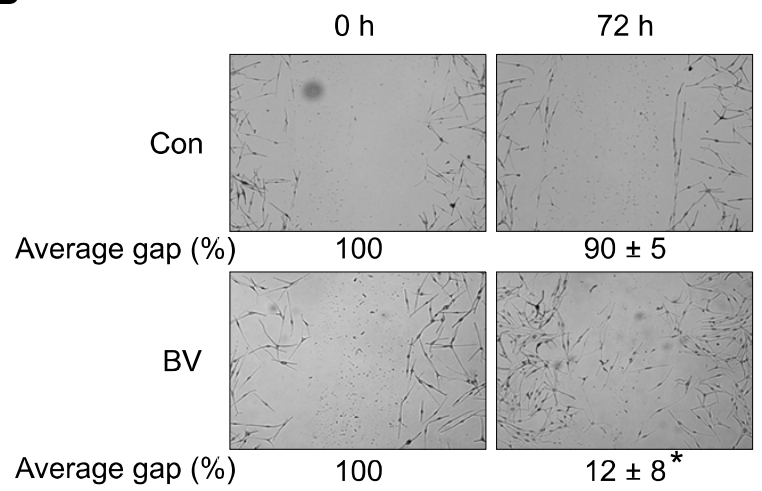

C

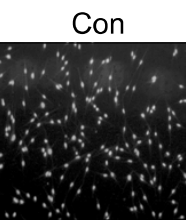

100

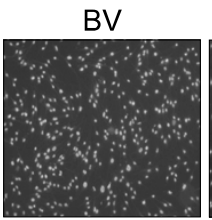

$250.9 \pm 15^{*}$
$A+B V$

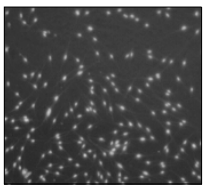

$117.9 \pm 10^{*}$

Figure 4. $\mathrm{BV}$ induces melanocyte dendricity and migration through $\mathrm{PLA}_{2}$ activation. (A) The image of melanocytes was captured after 3 days in control (Con), $10 \mu \mathrm{g} / \mathrm{ml}$ of BV (BV), or BV plus a PLA2 inhibitor, aristolochic acid (A + BV) treated cells. The number of melanocytes presenting more than two dendricities was counted on days 1,3 and 5 in melanocytes. The results represent the averaged results of four individual cultures. The error bars indicate S.E. $(n=4)$. (B) Area of the scratch repopulated by melanocytes (average gap) after 3 days of incubation in $10 \mu \mathrm{g} / \mathrm{ml}$ of BV (BV) compared with control (Con). The results represent the averaged results of four individual cultures. Values are mean \pm S.E. of four individual experiments; ${ }^{*} P<0.01$. (C) Migration was measured by Boyden chamber invasion assay with $10 \mu \mathrm{g} / \mathrm{ml}$ of BV (BV) in the absence or presence of aristolochic acid (A + BV). The migrated cells were photographed and the quantitation was carried out as percentage migrated cells compared with the control. Values are mean \pm S.E. of three individual experiments; ${ }^{*} P<0.01$. 
which PI3K/Akt activation was investigated, a polyclonal antibody that only recognizes the phosphorylated form of Akt and a PI3K inhibitor, wortmannin were used. The phosphorylation of Akt was gradually increased until $4 \mathrm{~h}$ and lasted for 5 days after BV treatment (Figure $3 \mathrm{~A}$, middle), but in the presence of wortmannin, BV-induced Akt phosphorylation was completely suppressed in melanocytes (Figure 3B, middle), indicating that BV activates PI3K/Akt pathway in melanocytes.

Then, we examined that which signaling pathway may be involved in the melanocyte proliferation or melanogenesis by BV treatments. In the presence of U0126, wortmannin or H89, BVinduced melanocyte proliferation was partially inhibited (Figure 3C). These results suggest that $B V$ increased the number of melanocytes through the activation of PKA, ERK and PI3K/Akt.

U0126 inhibited tyrosinase expression even in the absence of BV and completely suppressed tyrosinase expression by BV (Figure 3D). BVinduced tyrosinase expression was also completely inhibited by $\mathrm{H} 89$, whereas wortmannin had no effect (Figure 3D). Taken together, our results suggest that $B V$ induces tyrosinase expression through PKA and ERK activation.

\section{BV stimulates melanocyte dendricity}

Recently, it has been reported that $\mathrm{SPLA}_{2}$, a component of BV induces melanocyte dendricity (Scott et al., 2006). To confirm the effect of BV on melanocyte dendricity, melanocytes were treated with $10 \mu \mathrm{g} / \mathrm{ml}$ of BV and dendricity was quantified 1,3 and 5 days later. Figure 4 shows representative images of cells treated with BV compared with control cells. The majority of control cells exhibited a typical bipolar morphology, whereas BV-treated cells had multiple dendrites (Figure 4A, upper). Quantification of dendricity showed that BV increases the percentage of cells with more than two dendrites by $23 \%$ in a time dependent manner (Figure 4A, lower). Moreover, these BV-induced melanocyte dendricity was completely suppressed by pre-treatment of $\mathrm{SPLA}_{2}$ inhibitor, aristolochic acid (Figure 4A), suggesting that BV-induced melanocyte dendricity is through the $\mathrm{SLA}_{2}$ activation.

\section{BV stimulates melanocyte migration}

Previously, it has been reported that $\mathrm{SPLA}_{2}$, a component of BV induces vascular endothelial cell migration (Rizzo et al., 2000). Melanocyte migration is also an important factor for repigmenation of vitiligo skin. Thus, we examine whether BV induces melanocyte migration by using cell migration assays. Ten $\mu \mathrm{g} / \mathrm{ml}$ of BV was treated for 3 days. As shown in Figure 4B, BV stimulated significantly melanocyte migration and wound closure compared with control. Although $10 \mu \mathrm{g} / \mathrm{ml}$ of BV increased the number of melanocyte time dependently by 7 days, BV induced not much increment in melanocyte proliferation on day 3 (Figure 1B), indicating that the scratch areas were repopulated by migration rather than by increased proliferation. To confirm the effect of BV on the cell migration, Boyden chamber assays were performed. It also showed an increase in the number of migrated cells by two-fold in the presence of BV in the lower chamber as compared with controls (Figure 4C). However, the BV-induced melanocyte migration was completely suppressed by the treatment of $\mathrm{PLA}_{2}$ inhibitor, aristolochic acid (Figure 4C), suggesting that $\mathrm{BV}$-induced melanocyte migration is through the $\mathrm{PLA}_{2}$ activation.

\section{Discussion}

Proliferation, differentiation and migration of melanocytes and melanoblasts are regulated by keratinocyte-derived factors and some coat color genes. Any therapy for vitiligo must explain not only the repopulation of melanocytes but also their functional development. Vitiligo is an acquired pigmentary disorder characterized by depigmentation of skin and hair. As the pathogenesis of this disease is still obscure, the treatment of vitiligo has generally been unsatisfactory and often disappointing. In this study, we demonstrated that BV induces human melanocyte proliferation, melanogenesis, dendricity and migration.

In this study, we demonstrated that treatment with BV causes a dose-dependent increase in melanocyte cell number as much as forskolin did and this proliferation lasted for 7 days (Figure 1B). We also examined that BV induces the level of cAMP in melanocytes. Similar to other agents that increase intracellular cAMP (Eisinger and Marko, 1982; Halaban et al., 1986; Abdel-Malek et al., 1992), forskolin enhances the proliferation of human melanocytes. Although BV increased melanocyte proliferation as much as forskolin did, the increased level of cAMP was $10 \%$ of that induced by forskolin (Table 1). These results imply that besides an increased level of cAMP, other factors may be involved in the BV-induced melanocyte proliferation. In fact, our studies showed P13K/Akt activation by BV treatment besides CREB and ERK activation which can be induced by increased cAMP. Moreover, in melanocytes, a single growth factor may activate the ERK cascade but is not 
able to sustain melanocyte proliferation. A combination of at least two growth factors is needed to induce melanocyte proliferation (Moellmann and Halaban, 1998). We also demonstrated that the activation of PKA, ERK and PI3K/Akt are needed for the $B V$-induced melanocyte proliferation. Actually, BV is a rich source of pharmacologically active peptides, including melittin (a major component of $B V)$, secreted phospholipase A2 (sPLA $A_{2}$, apamin, adolapin, and mast cell degranulating peptide (Lariviere and Melzack, 1996). Among them, sPLA $A_{2} X$ stimulates melanocyte dendricity and melanogenesis, but not melanocyte proliferation (Scott et al., 2006). We also examined the effect of melittin on the human melanocyte. However, it has little effect on the melanocyte proliferation (data not shown). Thus, these suggest that each component of BV synergistically induces melanocyte proliferation.

cAMP is a critical component of melanogenesis, which involves both melanin synthesis and movement of melanosomes to newly formed dendrites at the cell periphery (Busca and Ballotti, 2000). The rate-limiting enzyme in melanin synthesis is tyrosinase (Ito, 2003). In this study, we also demonstrated that BV induces melanogenesis in human melanocytes by increasing MITF-M and tyrosinase expression and a subsequent increase in the melanin content. BV-induced melanogenesis reached the maximum at $1.25 \mu \mathrm{g} / \mathrm{ml}$ of $B V$ but, even at the concentration of $10 \mu \mathrm{g} / \mathrm{ml}$, which was optimal for melanocyte proliferation, melanogenesis was also induced. These results imply that although the effect of BV may be different on the dosage, $10 \mu \mathrm{g} / \mathrm{ml}$ of BV has dual effect on the melanocyte proliferation as well as melanogenesis.

The role of CAMP in the signaling pathways regulating melanogenesis has been studied. Activation of PKA and CREB by elevated CAMP levels leads to production of MITF-M, a melanocytespecific transcription factor crucial for melanocyte development and differentiation (Hodgkinson et al., 1993) and the increased MITF-M activate tyrosinase gene expression (Busca and Ballotti, 2000). However, cAMP-dependent and PKA-independent pathways have also been linked to the regulation of melanogenesis, including the Ras/ERK pathways (Busca and Ballotti, 2000). In the present study, we also examined that BV-induced tyrosinase expression is dependent on the PKA and MEK/ERK pathways not PI3K/Akt. These results suggest that $B V$ may induce melanogenesis through cAMP-dependent two pathways; PKAdependent and PKA-independent pathways.

BV also activated the PI3K/Akt pathway in human melanocytes; however, this pathway influ- enced melanocyte proliferation, not melanogenesis. The inhibitor of PI3K or its target, p70S6 kinase, markedly stimulates melanogenesis in melanoma cells, thus mimicking the action of CAMP elevating agents (Busca et al., 1996). In concert, when human melanocytes were treated with an inhibitor of PI3K, wortmannin, the basal level of tyrosinase was slightly increased (Figure 3D). However, BV-induced tyrosinase expression was not suppressed by wortmannin, supporting previous report that forskolin partially inhibits PI3K activity and phosphorylation (Busca et al., 1996).

Melanocyte dendricity, or dendrite outgrowth, constitutes a very important feature characterizing melanocyte activation, and it is an essential process to ensure melanin transfer to keratinocyte. In this study, we demonstrated that $B V$ stimulates melanocyte dendricity through $\mathrm{PLA}_{2}$ activation. This is in concert with a recent report that secreted $\mathrm{PLA}_{2}-\mathrm{X}$, a component of BV induces melanocyte dendricity (Scott et al., 2006).

In this study, we also demonstrated that BV induces melanocyte migration through $\mathrm{PLA}_{2}$ activation. Melanocyte migration from the neighboring normal epidermis or hair bulb palys an important role in repigmenation of vitiligo skin. In fact, topical tacrolimus (FK506), a potential therapeutic option for vitiligo, promotes cell migration and tyrosinase activation in human melanocytes (Kang and Choi, 2006), implying that BV has a potential therapeutic drug for vitiligo.

In summary, we demonstrated that BV exerts positive effects on melanocyte proliferation, melanogenesis, dendricity and migration through complex signaling pathways in vitro. This implies that BV may be developed as a therapeutic drug for inducing repigmentation in vitiligo skin.

\section{Acknowledgments}

The authors thank Dr. Christopher MH Kim at Pochon Cha University for kind donation of bee venom. This research was supported by a grant (code SC3270) from Stem Cell Research Center of the 21st Century Frontier Research Program funded by the Ministry of Science and Technology, Republic of Korea.

\section{References}

Abdel-Malek Z, Swope VB, Pallas J, Krug K, Nordlund JJ. Mitogenic, melanogenic, and cAMP responses of cultured neonatal human melanocytes to commonly used mitogens. J Cell Physiol 1992;150:416-25

Akdis CA, Akdis M, BleskinT, Wymann D, Alkan SS, Muller $\mathrm{U}$, Blaser K. Epitope-specific T cell tolerance to phospholipase A2 in bee venom immunotherapy. J Clin Invest 


\section{6;98:1676-83}

Arita Y, ODriscoll KR, Weinstein IB. Growth of human melanocyte cultures supported by 12-O-tetradecanoylphorbol-13-acetate is mediated through protein kinase $C$ activation. Cancer Res 1992;52:4514-21

Bertolotto C, Busca R, Abbe P, Bille K, Aberdam E, Ortonne JP, Ballotti R. Different cis-acting elements are involved in the regulation of TRP1 and TRP2 promoter activities by cyclic AMP: pivotal role of $M$ boxes (GTCATGTGCT) and of microphthalmia. Mol Cell Biol 1998;18:694-702

Busca R, Bertolotto C, Ortonne JP, Ballotti R. Inhibition of the phosphatidylinositol 3 kinase/p70(S6)-kinase pathway induces B16 melanoma cell differentiation. J Biol Chem 1996;271:31824-30

Busca R, Ballotti R. Cyclic AMP a key messenger in the regulation of skin pigmentation. Pigment Cell Res 2000;13: 60-9

Eiseman JL, VonBredow J, Alvares AP. Effect of honeybee (Apis mellifera) venom on the course of adjuvant-induced arthritis and depression of drug metabolism in the rat. Biochem Pharmacol 1982;31:1139-46

Eisinger M, Marko O. Selective proliferation of normal human melanocytes in vitro in the presence of phorbol ester and cholera toxin. Proc Natl Acad Sci USA 1982;79:2018-22

Englaro W, Rezzonico R, Durand-Clement M, Lallemand D, Ortonne JP, Ballotti R. Mitogen-activated protein kinase pathway and AP-1 are activated during cAMP-induced melanogenesis in B16 melanoma cells. J Biol Chem 1995;270:24315-20

Grabbe J, Welker P, Dippel E, Czarnetzki BM. Stem cell factor, a novel cutaneous growth factor for mast cells and melanocytes. Arch Dermatol Res 1994;287:78-84

Halaban R, Ghosh S, Duray P, Kirkwood JM, Lerner AB. Human melanocytes cultured from nevi and melanomas. J Invest Dermatol 1986;87:95-101

Halaban R, Ghosh S, Baird A. bFGF is the putative natural growth factor for human melanocytes. In Vitro Cell and Dvl Biol 1987;23:47-52

Hirobe T, Furuya R, Ifuku O, Osawa M, Nishikawa S. Granulocyte-macrophage colony-stimulating factor is a keratinocyte-derived factor involved in regulating the proliferation and differentiation of neonatal mouse epidermal melanocytes in culture. Exp Cell Res 2004;297:593-606

Hodgkinson CA, Moore KJ, Nakayama A, Steingrimsson E, Copeland NG, Jenkins NA, Arnheiter H. Mutations at the mouse microphthalmia locus are associated with defects in a gene encoding a novel basic-helix-loop-helix-zipper protein. Cell 1993;74:395-404

Imokawa G. Autocrine and paracrine regulation of melanocytes in human skin and in pigmentary disorders. Pigment Cell Res 2004;17:96-110

Ito. The IFPCS presidential lecture: a chemist's view of melanogenesis. Pigment Cell Res 2003;16:230-6

Jimbow K, Roth SI, Fitzpatrick TB, Szabo G. Mitotic activity in non-neoplastic melanocytes in vivo as determined by histochemical, autoradiographic, and electron microscope studies. J Cell Biol 1975;66:663-70

Kang HY, Choi YM. FK506 increases pigmentation and migration of human melanocytes. $\mathrm{Br} \mathrm{J}$ Dermatol 2006; 155:1037-40

Kim HW, Kwon YB, Ham TW, Rho DH, Yoon SY, Lee HJ, Han HJ, Yang IS, Beitz AJ, Lee, JH. Acupoint stimulation using bee venom attenuates formalin-induced pain behavior and spinal cord fos expression in rats. J Vet Med Sci 2003; 65:349-55

Kimura K, Hattori S, Kabuyama Y, Shizawa Y, Takayanagi J, Nakamura S, Toki S, Matsuda Y, Onodera K, Fukui Y. Neurite outgrowth of PC12 cells is suppressed by wortmannin, a specific inhibitor of phosphatidylinositol 3-kinase. J Biol Chem 1994:269:18961-7

Kovac SO. Vitilogo. J Am Acad Dermatol 1998;38:647-66

Krasagakis K, Garbe C, Kruger-Krasagakes S, Orfanos CE. 12-O-tetradecanoylphorbol-13-acetate not only modulates proliferation rates, but also alters antigen expression and LAK-cell susceptibility of normal human melanocytes in vitro. J Invest Dermtol 1993;100:653-9

Kwon YB, Lee JD, Lee HJ, Han HJ, Mar WC, Kang SK, Beitz AJ, Lee JH. Bee venom injection into an acupuncture point reduces arthritis-associated edema and nociceptive responses. Pain 2001;90:271-80

Lan CC, Chen GS, Chiou MH, Wu CS, Chang CH, Yu HS. FK506 promotes melanocyte and melanoblast growth and creates a favorable milieu for cell migration via keratinocytes: possible mechanisms of how tacrolimus ointment induces repigmentation in patients with vitiligo. $\mathrm{Br} \mathrm{J}$ Dermatol 2005; 153:498-505

Lariviere WR, Melzack R. The bee venom test: a new tonic-pain test. Pain 1996;66:271-7

Lee JD, Kim SY, Kim TW, Lee SH, Yang HI, Lee YH. Antiinflammatory effect of bee venom on type II collagen-induced arthritis. Ame J Chin Med 2004;32:361-7

Maeda K, Tomita Y, Naganuma M, Tagami H. Phospholipases induce melanogenesis in organ-cultures skin. Photochem Photobiol 1996;64:220-3

Maeda K, Naganuma M. Melanocyte-stimulating properties of secretory phospholipase A2. Photochem Photobiol 1997;65:145-9

Medrano EE, Yang F, Boissy R, Farooqui J, Shah V, Matsumoto K, Nordlund JJ, Park HY. Terminal differentiation and senescence in the human melanocyte: repression of tyrosine-phosphorylation of the extracellular signal-regulated kinase 2 selectively defines the two phenotypes. Mol Cell Biol 1994;5:497-509

Moellmann g, Halaban R. Growth factor receptors and signal transduction regulating the proliferation and differentiation of melanocytes. In The Pigmentatary System : Physiology and Pathophysiology (Nordlund JJ, Biossy RE, Hearing VJ, King RA, eds). New York: N Engl J Med 1998;328:1302-7

Oka M, Ichihashi M, Chakraborty AK. Enhanced expression of protein kinase $\mathrm{C}$ subspecies in melanogenic compartments in B16 melanoma cells by UVB or MSH. J Invest 
Dermatol 1996;106:377-8

Park MS, Park HL, Kim WH, Cho HS, Lee JH. Presence of autocrine hepatocyte growth factor-Met signaling and its role in proliferation and migration of SNU-484 gastric cancer cell line. Exp Mol Med 2005;37:213-9

Pawelek JM. Evidence suggesting that a cyclic AMP-dependent protein kinase is a positive regulator of proliferation in Cloudman S91 melanoma cells. J Cell Physiol 1979; 98:619-25

Rizzo MT, Nguyen E, Aldo-Benson M, Lambeau G. Secreted phospholipase $A(2)$ induces vascular endothelial cell migration. Blood 2000;96:3809-15

Robinson MJ, Cobb MH. Mitogen-activated protein kinase pathways. Curr Opin Cell Biol 1997;9:180-6

Scott GA, Jacobs SE, Pentland AP. sPLA2-X stimulates cutaneous melanocyte dendricity and pigmentation through a lysophosphatidylcholine-dependent mechanism. J Invest Dermatol 2006;126:855-61

Swope VB, Medrano EE, Smalara D, Abdel-Malek ZA. Long-term proliferation of human melanocytes is supported by the physiologic mitogens alpha-melanotropin, endothelin-1, and basic fibroblast growth factor. Exp Cell Res 1995;217:453-9

Tada A, Pereira E, Beitner-Johnson D, Kavanagh R, AbdelMalek ZA. Mitogen- and ultraviolet-B-induced signaling pathways in normal human melanocytes. J Invest Dermatol 2002;118:316-22

Wilkins L, Gilchrest BA, Szabo G, Weinstein R, Maciag T. The stimulation of normal human melanocyte proliferation in vitro by melanocyte growth factor from bovine brain. J Cell Physiol $1985 ; 122: 350-61$ 